\title{
Rank-one convexity and polyconvexity of Hencky-type energies
}

\author{
Patrizio Neff ${ }^{1, *}$, Ionel-Dumitrel Ghiba ${ }^{1,2}$, Johannes Lankeit ${ }^{3}$, and Robert Martin ${ }^{1}$ \\ ${ }^{1}$ Lehrstuhl für Nichtlineare Analysis und Modellierung, Fakultät für Mathematik, Universität Duisburg-Essen, \\ Thea-Leymann Str. 9, 45127 Essen, Germany \\ 2 Alexandru Ioan Cuza University of Iaşi, Department of Mathematics, Blvd. Carol I, no. 11, 700506 Iaşi, Romania. \\ ${ }^{3}$ Institut für Mathematik, Universität Paderborn, Warburger Str. 100, 33098 Paderborn, Germany
}

\begin{abstract}
We investigate a family of isotropic volumetric-isochorically decoupled strain energies based on the Hencky-logarithmic (true, natural) strain tensor $\log U$. The main result of this note is that for $n=2$ the considered energies are rank-one convex for suitable values of two material parameters. We also conjecture that there are values of the material parameters such that the corresponding energies are polyconvex.
\end{abstract}

(C) 2014 Wiley-VCH Verlag GmbH \& Co. KGaA, Weinheim

\section{Introduction}

In a recent contribution [7], we have introduced a family of nonlinear elastic energies based on certain invariants of the Hencky tensor $\log U$, namely $\left\|\operatorname{dev}_{n} \log U\right\|^{2}$ and $(\operatorname{tr}(\log U))^{2}$, where $F=\nabla \varphi$ is the deformation gradient, $U=\sqrt{F^{T} F}$ is the right stretch tensor, $\log U$ is the referential (Lagrangian) logarithmic strain tensor, and $\operatorname{dev}_{n} X=X-\frac{1}{n} \operatorname{tr}(X) \cdot \mathbb{1}$ is the deviatoric part (the projection onto the traceless tensors) of the second order tensor $X \in \mathbb{R}^{n \times n}$ and $\|\cdot\|$ is the Frobenius tensor norm. The considered family of exponentiated Hencky-logarithmic strain type energies is given by

$$
W_{e H}(F):= \begin{cases}\underbrace{\frac{\mu}{k} e^{k\|\operatorname{dev} n \log U\|^{2}}+\frac{\kappa}{2 \widehat{k}} e^{\widehat{k}(\operatorname{tr}(\log U))^{2}}}_{\text {volumetric-isochoric split }} & \text { if } \quad \operatorname{det} F>0, \\ +\infty & \text { if } \quad \operatorname{det} F \leq 0,\end{cases}
$$

where $\mu>0$ is the shear (distortional) modulus, $\kappa=\frac{2 \mu+3 \lambda}{3}>0$ is the bulk modulus with the first Lamé constant $\lambda$, and $k, \widehat{k}$ are dimensionless parameters. The immediate importance of the family (1) of free-energy functions is seen by looking at small (but not infinitesimally small) strains. For small elastic strains, $W_{e H}$ approximates the classical quadratic Hencky strain energy $W_{H}(F):=\mu\left\|\operatorname{dev}_{n} \log U\right\|^{2}+\frac{\kappa}{2}[\operatorname{tr}(\log U)]^{2}$, which is not rank-one convex. The Hencky energy $W_{H}$ has been introduced by Heinrich Hencky starting from 1928 [4] and has since then acquired a unique status in finite elastostatics and especially in finite strain elasto-plasticity. The first axiomatic study on the nonlinear stress-strain function involving a logarithmic strain tensor is, however, due to the famous geologist George Ferdinand Becker in 1893 [9].

In plane elastostatics, i.e. $n=2$, we prove the rank-one convexity of the proposed family $W_{e H}$ for $k \geq \frac{1}{4}$ and $\hat{k} \geq \frac{1}{8}$. Moreover, in [7] we show that the corresponding Cauchy (true) stress - true strain relation is invertible for $n=2,3$ and we discuss the monotonicity of the Cauchy stress tensor as a function of the true strain tensor. We also prove that the rankone convexity of the energies belonging to this family is not preserved in dimension $n=3$ and that the energy $W(F):=$ $e^{k\|\log U\|^{2}}, F \in \mathrm{GL}^{+}(n), n \in \mathbb{N}, n \geq 2$, is not rank-one convex. An immediate application of $W_{e H}$ in multiplicative elasto-plasticity $[3,11]$ is also proposed in [7].

\section{Rank-one convexity in plane elastostatics}

In order to study the rank-one convexity of the family of energies $W_{e H}$, the first step is to check if the volumetric response $\operatorname{det} F \mapsto e^{\hat{k}(\log \operatorname{det} F)^{m}}$ is convex. In arbitrary dimensions, the following result holds:

Proposition 2.1 The function $\operatorname{det} F \mapsto e^{\hat{k}(\log \operatorname{det} F)^{m}}, F \in \mathrm{GL}^{+}(n)$, is convex in $\operatorname{det} F$ for $\hat{k} \geq \frac{1}{m^{(m+1)}}$.

The next step is to prove the rank-one convexity of the isochoric exponentiated Hencky energy $F \mapsto e^{k\left\|\operatorname{dev}_{2} \log U\right\|^{2}}$ in plane elastostatics. We show that although $F \mapsto\left\|\operatorname{dev}_{2} \log U\right\|^{2}$ is not rank-one convex, the function $F \mapsto e^{\left\|\operatorname{dev}_{2} \log U\right\|^{2}}$ is indeed rank-one convex. In terms of the singular values $\lambda_{1}, \lambda_{2}$ of the right stretch tensor $U=\sqrt{F^{T} F}$, we have the following representation of the isochoric exponentiated Hencky energy:

* Corresponding author: e-mail patrizio.neff@uni-due.de 


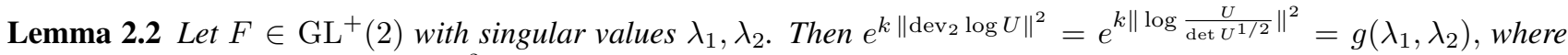
$g: \mathbb{R}_{+}^{2} \rightarrow \mathbb{R}, g\left(\lambda_{1}, \lambda_{2}\right):=e^{\frac{k}{2}\left(\log \frac{\lambda_{1}}{\lambda_{2}}\right)^{2}}$.

Using this representation and the necessary and sufficient criteria for rank-one convexity given by Knowles and Sternberg [1] we establish the following result:

Proposition 2.3 If $k \geq \frac{1}{4}$, then the function $F \mapsto e^{k\left\|\operatorname{dev}_{2} \log U\right\|^{2}}$ is rank-one convex in $\mathrm{GL}^{+}(2)$.

In view of the above auxiliary results we conclude:

Theorem 2.4 (planar rank-one convexity) The functions $W_{e H}: \mathbb{R}^{n \times n} \rightarrow \overline{\mathbb{R}}_{+}$from the family of exponentiated Hencky type energies $W_{e H}(F)$ are rank-one convex for the two-dimensional situation $n=2$, for $\mu>0, \kappa>0, k \geq \frac{1}{4}$ and $\widehat{k} \geq \frac{1}{8}$.

We give the conjecture:

Conjecture 2.5 (planar polyconvexity) The functions $W_{e H}: \mathbb{R}^{n \times n} \rightarrow \overline{\mathbb{R}}_{+}$from the family of exponentiated Hencky type energies $W_{e H}(F)$ are polyconvex for the two-dimensional situation $n=2$, for $\mu>0, \kappa>0, k \geq \frac{1}{4}$ and $\widehat{k} \geq \frac{1}{8}$.

In plane elasto-statics, the rank-one convex energy $W_{e H}(F)$ is applicable to the bending or shear of long strips and to all cases in which symmetry arguments can be applied to reduce the formulation to a planar deformation.

\section{Outlook for three dimensions and conclusions}

In [7] it is also shown that for all parameters $k>0$ the energy function $F \mapsto \frac{\mu}{k} e^{k\left\|\operatorname{dev}_{3} \log U\right\|^{2}}, F \in \mathrm{GL}^{+}(3)$, is not rank-one convex. Numerical tests suggest that the rank-one convexity domain of the distortional energy function $F \mapsto$ $\frac{\mu}{k} e^{k\left\|\operatorname{dev}_{3} \log U\right\|^{2}}, F \in \mathrm{GL}^{+}(3)$, is a large cone $\left\{U \in \operatorname{PSym}(3) \mid\left\|\operatorname{dev}_{3} \log U\right\|^{2}<27\right\}$, while $k \geq \frac{3}{16}$ is the necessary condition for separate convexity of $e^{k\left\|\operatorname{dev}_{3} \log U\right\|^{2}}$ in the three-dimensional situation $n=3$.

By purely differential geometric reasoning, in forthcoming papers $[5,6,10]$ it will be shown that

$$
\begin{aligned}
& \operatorname{dist}_{\text {geod }}^{2}\left((\operatorname{det} F)^{1 / n} \cdot \mathbb{1}, \mathrm{SO}(n)\right)=\operatorname{dist}_{\text {geod }, \mathbb{R}_{+} \cdot \mathbb{1}}^{2}\left((\operatorname{det} F)^{1 / n} \cdot \mathbb{1}, \mathbb{1}\right)=|\log \operatorname{det} F|^{2}, \\
& \operatorname{dist}_{\text {geod }}^{2}\left(\frac{F}{(\operatorname{det} F)^{1 / n}}, \mathrm{SO}(n)\right)=\operatorname{dist}_{\text {geod, SL }(\mathrm{n})}^{2}\left(\frac{F}{(\operatorname{det} F)^{1 / n}}, \mathrm{SO}(n)\right)=\left\|\operatorname{dev}_{n} \log U\right\|^{2},
\end{aligned}
$$

where dist ${ }_{\text {geod }}^{2}$ is the canonical left invariant geodesic distance on the Lie-groups $\operatorname{GL}(n), \operatorname{SL}(n)$ and $\mathbb{R}_{+} \cdot \mathbb{1}$, see also [2,10]. Hence, using this terminology, in the present note we have shown, for $\mu>0, \kappa>0, k \geq \frac{1}{4}$ and $\widehat{k} \geq \frac{1}{8}$, the rank-one convexity of $W_{e H}(F):=\frac{\mu}{k} e^{k \operatorname{dist}_{\text {geod,SL }(2)}^{2}\left(\frac{F}{\operatorname{det} F^{1 / 2}}, \mathrm{SO}(2)\right)}+\frac{\kappa}{2 \widehat{k}} e^{\widehat{k} \operatorname{dist}_{\text {geod, } \mathbb{R}_{+} \cdot \mathbb{1}}^{2}\left(\operatorname{det} F^{1 / 2} \cdot \mathbb{1}, \mathrm{SO}(2)\right)}$. In a forthcoming paper [8] we will provide a detailed study of the polyconvexity of the energies $W_{e H}$.

\section{References}

[1] J.K. Knowles and E. Sternberg. On the ellipticity of the equations of nonlinear elastostatics for a special material. J. Elasticity, 5(3-4):341-361, 1975.

[2] J. Lankeit, P. Neff, and Y. Nakatsukasa. The minimization of matrix logarithms: On a fundamental property of the unitary polar factor. Linear Algebra and its Applications, 449(0):28 - 42, 2014.

[3] P. Neff, K. Chełmiński, and H.D. Alber. Notes on strain gradient plasticity. Finite strain covariant modelling and global existence in the infinitesimal rate-independent case. Math. Mod. Meth. Appl. Sci. (M3AS), 19(2):1-40, 2009.

[4] P. Neff, B. Eidel, and R. Martin. The axiomatic deduction of the quadratic Hencky strain energy by Heinrich Hencky. arXiv:1402.4027, 2014.

[5] P. Neff, B. Eidel, F. Osterbrink, and R. Martin. A Riemannian approach to strain measures in nonlinear elasticity. to appear in C. R. Acad. Sci., 2014.

[6] P. Neff, B. Eidel, F. Osterbrink, and R. Martin. Appropriate strain measures: The Hencky shear strain energy $\left\|\operatorname{dev} \log \sqrt{F^{T} F}\right\|^{2}$ measures the geodesic distance of the isochoric part of the deformation gradient $\bar{F} \in \mathrm{SL}(3)$ to $\mathrm{SO}(3)$ in the canonical left invariant Riemannian metric on SL(3). in preparation, 2014.

[7] P. Neff, I.D. Ghiba, and J. Lankeit. Rank one convexity of the exponentiated Hencky-logarithmic strain energy in finite elastostatics. Preprint arXiv:1403.3843, 2014.

[8] P. Neff, I.D. Ghiba, J. Lankeit, R. Martin, and D. Steigmann. Polyconvexity of the exponentiated Hencky-logarithmic strain energy in plane finite elastostatics. in preparation, 2014.

[9] P. Neff, I. Münch, and R. Martin. Rediscovering G.F. Becker's early axiomatic deduction of a multiaxial nonlinear stress-strain relation based on logarithmic strain. arXiv:1403.4675, 2014.

[10] P. Neff, Y. Nakatsukasa, and A. Fischle. A logarithmic minimization property of the unitary polar factor in the spectral norm and the Frobenius matrix norm. to appear in SIAM J. Matrix Analysis, 2014.

[11] P. Neff and C. Wieners. Comparison of models for finite plasticity. A numerical study. Comput. Visual. Sci., 6:23-35, 2003. 